\title{
Comparison of Survey Sampling Methods for Estimation of Vaccination Coverage in an Urban Setup of Assam, India
}

\author{
Dilip C. Nath*, Bhushita Patowari \\ Department of Statistics, Gauhati University, Guwahati, India \\ Email: "dilipc.nath@gmail.com, pbhushita@yahoo.com
}

Received 29 September 2015; accepted 20 November 2015; published 23 November 2015

Copyright (C) 2015 by authors and Scientific Research Publishing Inc.

This work is licensed under the Creative Commons Attribution International License (CC BY). http://creativecommons.org/licenses/by/4.0/

(c) () D Den Access

\begin{abstract}
Background: Immunization averts a large number of children in each year. The burden of vaccine preventable diseases remains high in developing countries compared to developed countries. To overcome from this burden different types of immunization programs have been implemented. For better immunization coverage in developing countries, considerable progress is to be made to improve the knowledge and awareness regarding importance of vaccines. In this study a comparative study of immunization coverage under two sampling methods has been performed. Methods: In this study variance and design effect of proportion of children vaccinated against different types of vaccines (BCG, OPV, DPT, Hepatitis B, Hib, Measles and MMR) are estimated under two stage $(30 \times 30)$ cluster and systematic sampling for comparison of these two survey sampling methods. Also the homogeneity of clusters has been tested by using chi-square test. Results: It is observed that BCG, OPV and DPT vaccination coverage is more than $90 \%$ whereas Hepatitis B, Measles, Hib and MMR vaccination coverage is between $50 \%-64 \%$ only. Here systematic random sampling is more complicated than two stage $(30 \times 30)$ cluster sampling. Also the result shows that the clusters are homogeneous with respect to proportion of children vaccinated. Conclusion: There is no significant difference between the two survey methodologies regarding the point estimation of vaccination coverage but estimation of variances of vaccination coverage is less in two stage $(30 \times 30)$ cluster sampling than that of the systematic sampling. Also the clusters are homogeneous. Very less improvement has been observed in case of fully vaccination coverage than the previous study. From the study it can be said that two stage $(30 \times 30)$ cluster sampling will be preferred to systematic sampling and simple random sampling method.
\end{abstract}

\section{Keywords}

Vaccine Coverage, Cluster Sampling, Systematic Sampling, Design Effect, Marascuilo Procedure

${ }^{*}$ Corresponding author. 


\section{Introduction}

World Health Organization (WHO) recommends that all children should receive one dose of Bacillis CalmetteGuerin Vaccine (BCG), three doses of diphtheria-tetanus-pertusis vaccine (DPT), three doses of either oral polio vaccine (OPV) or inactivated polio vaccine (IPV), three doses of hepatitis B vaccine, and one dose of a measles virus-containing vaccine (MVCV), either anti-measles alone or in combination with other antigens. It also recommends three doses of vaccine against infection with Haemophilus influenza type b (Hib). To boost immunity at older ages, additional immunizations are recommended for healthcare workers, travelers, high-risk groups and people in areas where the risk of specific vaccine-preventable diseases is high [1]. The important role played by the WHO's EPI (Expanded Programme on Immunization) Cluster Survey in the success of national immunization programme efforts in many countries is widely recognized. The programme monitoring capability provided through the conduct of periodic cluster surveys has been especially important in developing country settings, where administrative records are often incomplete [2]. Together with EPI sampling other survey sampling has been compared in different studies [3]-[5]. According to WHO coverage of BCG vaccine is $87 \%$, DPT3 vaccine is $72 \%$ and OPV3 vaccine is 70\% in 2011 [6]. In a study Phukan et al. reported that the children of Assam in the North-East Region of India have consistently evidenced low rates for routine childhood immunizations. About $62.2 \%$ of the children were fully immunized [7]. Children are considered fully immunized if they receive one dose of BCG, three doses of OPV and DPT each and one dose of measles vaccine before reaching one year of age.

In this study estimates of vaccination coverage have been compared using design effect and variance of estimated proportion of children vaccinated against BCG, OPV, DPT, Hepatitis B, Hib, Measles and MMR (measles mumps rubella) vaccines under two stage $(30 \times 30)$ cluster sampling and systematic random sampling.

\section{Methods}

The data that has been used in this study is taken from a survey "Comparison of Two Survey Methodologies to Estimate Total Vaccination Coverage” sponsored by Indian Council of Medical Research (ICMR), New Delhi. It has been collected during the period from January to October, 2011 using following sampling techniques.

Two stage $(30 \times 30)$ cluster sampling: In this method the population needs to be divided into a complete set of non-overlapping subpopulations, usually defined by geographic or political boundaries. These subpopulations are called clusters. In the first stage, 30 of these clusters are sampled with probability proportionate to the size (PPS) of the population in the cluster. Sampling with probability proportionate to size allows the larger clusters to have a greater chance of being selected. The clusters are sampled without replacement. In the second stage of sampling, thirty subjects are selected within each cluster. Although the sampling unit is the individual subject, the sampling is conducted on the household level. Cluster sampling is often a practical approach to surveys because it samples by groups (clusters) of elements rather than by individual elements. It simplifies the task of constructing sampling frames, and it reduces the survey costs [8]. The advantages of two stage $(30 \times 30)$ cluster sampling over other designs are same as cluster sampling. A sampling frame listing all elements in the population may be impossible or costly to obtain, whereas to obtain a list of all clusters may be easy. Also the cost of obtaining data may be inflated by travel cost if the sampled elements are spread over a large geographic area.

Systematic random sampling: Systematic sampling is a random method of sampling in which only the first unit is selected with the help of random numbers and the rest get selected automatically according to some pre-designed pattern. If the population size $N=n k$, where $\mathrm{n}$ is the sample size and $k$ is an integer, and a random number less than or equal to $k$ be selected and every $k^{\text {th }}$ unit thereafter. This procedure is linear systematic sampling. When $N \neq n k$ then every $k^{\text {th }}$ unit be included in a circular manner till the whole list is exhausted, it is called circular systematic sampling. Systematic sampling is commonly used as an alternative to simple random sampling (SRS) because of its simplicity. It selects every $k^{\text {th }}$ element after a random start (between 1 and $\mathrm{k}$ ). Its procedural tasks are simple, and the process can easily be checked, whereas it is difficult to verify SRS by examining the results. It is often used in the final stage of multistage sampling when the fieldworker is instructed to select a predetermined proportion of units from the listing of dwellings in a street block. The systematic sampling procedure assigns each element in a population the same probability of being selected [8].

With the two stage $(30 \times 30)$ cluster sampling method in the first stage 30 wards are selected and in the second stage 30 units from each ward are selected. For the selection of second stage units in a selected ward only the first household is randomly selected. After the first household is visited, the surveyor moves to the "next" 
household, which is defined as the one whose front door is closest to the one just visited. Where there are bylane in a particular lane survey procedure is carried out in that place according to the serial household number in that bylane. This process continues until all 30 eligible subjects are found. The subjects are chosen by selecting a household and for more than one eligible subject (children from 6 months to 5 years of age) in a household all are selected.

After completing the $1^{\text {st }}$ sampling method (that is two stage $(30 \times 30)$ cluster sampling) in a ward, $2^{\text {nd }}$ sampling method (systematic random sampling) is carried out in same ward. In this sampling technique a random number is selected from random number table on the basis of the number of household in a lane where the survey was carried out in case of two stage $(30 \times 30)$ cluster sampling and this became the first sampling unit (household) of the systematic random sampling. After that each household is selected at an interval of 10 household and continuing the process until the 30 sampling units are not completed. Here the interval of household is taken as 10 so that the interval is neither too small nor too large. If we take the interval too small then we should get so many repetitions of the samples from two stage $(30 \times 30)$ cluster sampling which results same sampling unit in the $2^{\text {nd }}$ sampling method (systematic sampling) and if we take the interval too large then there should not be any similarity between the two sampling methodologies as the larger interval will cover larger area and both the sampling techniques would take different places.

\section{Statistical Analysis}

Analysis has been carried out in the following two sections.

\subsection{Section A}

Here, variance of proportion of vaccination coverage and design effect of the same has been estimated.

Let, $P=$ proportion of children who are vaccinated

Since same number of children has sampled per cluster, estimate of $P(\hat{P})$ is given by

$$
\hat{P}=\sum_{i=1}^{n=30} \frac{p_{i}}{n}
$$

where $p_{i}=$ the proportion of surveyed children in $i^{\text {th }}$ cluster

$n=$ the number of clusters

Then approximate estimated variance of $\hat{P}_{c}$ under cluster sampling [4] is given by

$$
\hat{v}\left(\hat{P}_{c}\right)=\sum_{i=1}^{n}\left(p_{i}-\hat{P}\right)^{2} /[n(n-1)]
$$

Again the estimated variance of $\hat{P}_{s y}$ under systematic sampling [9] is

$$
\hat{v}\left(\hat{P}_{s y}\right)=\left(\frac{N-n}{N}\right) \frac{\hat{p}(1-\hat{p})}{n-1}
$$

An approximate $95 \%$ confidence interval on $P$ can be obtained by using

$$
\hat{P} \pm 1.96 \sqrt{v(\hat{P})}
$$

The design effect may be estimated as

$$
\operatorname{deff}=\frac{\hat{v}(\text { esimated proportion under specified sampling })}{\hat{v}\left(\hat{P}_{s}\right)}
$$

where

$$
\hat{v}\left(\hat{P}_{s}\right)=\hat{P}(1-\hat{P}) /\left[\left(\sum_{i=1}^{n=30} n_{i}\right)-1\right]
$$

is the estimated variance under simple random sampling [4].

Also the design effect for cluster sampling vs systematic sampling is obtained as 


$$
\operatorname{deff}=\frac{\hat{v}\left(\hat{P}_{c}\right)}{\hat{v}\left(\hat{P}_{s y}\right)}
$$

\subsection{Section B}

In this section homogeneity of clusters have been tested by using chi-square test. That is to test equality of proportion of children vaccinated in each clusters. The test procedure is carried out taking Hepatitis B (at birth) vaccine (two stage $(30 \times 30)$ cluster sampling).

The null hypothesis is that there are no significant differences among the proportions of children vaccinated against Hepatitis B (at birth) in each clusters.

$\mathrm{H}_{0}: P_{1}=P_{2}=\cdots=P_{30}$

Against the alternative that all the proportions are not equal.

$\mathrm{H}_{1}$ : Not all $P_{j}$ 's are equal (where $j=1,2, \cdots, 30$ )

The test statistic is

$$
\chi^{2}=\sum \frac{\left(f_{o}-f_{e}\right)^{2}}{f_{e}}
$$

where

$f_{o}=$ observed frequency in a particular cell of a $2 \times 30$ contingency table

$f_{e}=$ expected frequency in a particular cell if the null hypothesis is true

If the null hypothesis is true the proportions are all equal across the population. And rejecting the null hypothesis only allows to reach the conclusion that all proportions are not equal. But the test statistics does not give any information about proportions that differ. To identify the differences between proportions we will rely on a multiple comparison procedure. The Marascuilo procedure [10] enables us to make comparisons between all pairs of groups. In this procedure the absolute value of the pairwise difference between sample proportions has to be computed. The absolute values of these differences are the test statistics. For each pairwise comparison a critical value is computed as follows:

$$
C V_{i j}=\sqrt{\chi_{\alpha, k-1}^{2}} \sqrt{\frac{\bar{p}_{i}\left(1-\bar{p}_{i}\right)}{n_{i}}+\frac{\bar{p}_{j}\left(1-\bar{p}_{j}\right)}{n_{j}}}
$$

where $\alpha=$ level of significance, $k=$ number of clusters

To compare each of test statistics with the corresponding critical value a specific pair is significantly different if the absolute difference in the sample proportion $\left|p_{i}-p_{j}\right|$ is greater than its critical range.

\section{Results}

Table 1 gives estimated coverage of BCG (at birth), OPV (OPV1 at birth, OPV2 at 6 weeks, OPV3 at 10 weeks, OPV4 at 14 weeks, OPV5 at 15 - 18 months and OPV6 at 5 years), DPT (DPT1 at 6 weeks, DPT2 at 10 weeks, DPT3 at 14 weeks, DPT4 at 15 - 18 months and DPT5 at 5 years), Hepatitis B (HepB1 at birth and HepB2 at 6 weeks), Hib (Hib1 at 6 weeks, Hib2 at 10 weeks and Hib3 at 14 weeks), Measles (at 9 months) and MMR (at 15 - 18 months) vaccine with $95 \%$ confidence intervals under two stage cluster and systematic sampling. Coverage of BCG vaccine is $99 \%$, OPV and DPT vaccine coverage is more than $90 \%$ except for OPV6 and DPT5. But coverage of Hepatitis B, Hib, Measles and MMR vaccines are only between 50\% - 64\%. Though the individual vaccination coverage is high for BCG, OPV and DPT vaccine but fully vaccination coverage is only $63.52 \%$. Both the survey methods have given point estimates of vaccination coverage with less difference.

Estimated variance of proportion of vaccination coverage is given in Table 2. It is seen that variances are less in case of two stage cluster sampling than the systematic sampling for all the vaccines namely BCG, OPV, DPT, Hepatitis B, Hib, Measles and MMR that are considered in the study. So the interval estimation of vaccination coverage has given better estimate in case of two stage $(30 \times 30)$ cluster sampling than the systematic sampling with less standard error (SE). 
Table 1. Estimated coverage of vaccines under two stage cluster $(30 \times 30)$ and systematic sampling.

\begin{tabular}{cccccc}
\hline \multirow{2}{*}{ Vaccine } & & \multicolumn{2}{c}{ Two stage cluster $(30 \times 30)$} & \multicolumn{2}{c}{ Systematic sampling } \\
\cline { 3 - 6 } BCG & & Coverage estimate & $95 \%$ CI & Coverage estimate & $95 \%$ CI \\
& & 0.99 & $(0.98,0.99)$ & 0.99 & $(0.98,0.99)$ \\
& OPV1 & 0.99 & $(0.98,0.99)$ & 0.99 & $(0.98,0.99)$ \\
& OPV2 & 0.98 & $(0.97,0.98)$ & 0.99 & $(0.98,0.99)$ \\
& OPV3 & 0.98 & $(0.97,0.98)$ & 0.99 & $(0.98,0.99)$ \\
OPV & OPV4 & 0.97 & $(0.96,0.97)$ & 0.99 & $(0.98,0.99)$ \\
& OPV5 & 0.90 & $(0.89,0.90)$ & 0.89 & $(0.86,0.91)$ \\
& OPV6 & 0.54 & $(0.53,0.54)$ & 0.54 & $(0.50,0.57)$ \\
DPT & DPT1 & 0.98 & $(0.97,0.98)$ & 0.99 & $(0.98,0.99)$ \\
& DPT2 & 0.98 & $(0.97,0.98)$ & 0.99 & $(0.98,0.99)$ \\
& DPT3 & 0.97 & $(0.96,0.97)$ & 0.98 & $(0.98,0.99)$ \\
& DPT4 & 0.90 & $(0.89,0.90)$ & 0.90 & $(0.88,0.91)$ \\
& DPT5 & 0.52 & $(0.51,0.52)$ & 0.51 & $(0.47,0.54)$ \\
& HepB1 & 0.58 & $(0.57,0.58)$ & 0.56 & $(0.52,0.59)$ \\
Hepatitis B & HepB2 & 0.59 & $(0.58,0.59)$ & 0.56 & $(0.52,0.59)$ \\
& Hib1 & 0.57 & $(0.56,0.57)$ & 0.55 & $(0.51,0.58)$ \\
& Hib2 & 0.57 & $(0.56,0.57)$ & 0.55 & $(0.51,0.58)$ \\
& Hib3 & 0.57 & $(0.50,0.64)$ & 0.55 & $(0.51,0.58)$ \\
Measles & & $(0.63,0.64)$ & 0.64 & $(0.60,0.67)$ \\
& & 0.64 & $(0.51,0.52)$ & 0.50 & $(0.46,0.53)$ \\
\hline
\end{tabular}

Table 2. Estimated variance of proportion of vaccination coverage $(\hat{P})$.

\begin{tabular}{|c|c|c|c|}
\hline \multirow{2}{*}{\multicolumn{2}{|c|}{ Vaccines }} & \multicolumn{2}{|c|}{ Methodology } \\
\hline & & Two stage cluster $(30 \times 30)$ & Systematic sampling \\
\hline \multicolumn{2}{|c|}{ BCG } & $9.2009 \times 10^{-09}$ & $2.44173 \times 10^{-06}$ \\
\hline \multirow{7}{*}{ OPV } & OPV1 & $1.1947 \times 10^{-08}$ & $4.87257 \times 10^{-06}$ \\
\hline & OPV2 & $3.2958 \times 10^{-08}$ & $9.70164 \times 10^{-06}$ \\
\hline & OPV3 & $3.2134 \times 10^{-08}$ & $1.32949 \times 10^{-05}$ \\
\hline & OPV4 & $1.2785 \times 10^{-07}$ & $1.56768 \times 10^{-05}$ \\
\hline & OPV5 & $5.4684 \times 10^{-06}$ & $2.29435 \times 10^{-04}$ \\
\hline & OPV6 & $6.0007 \times 10^{-07}$ & $1.06516 \times 10^{-04}$ \\
\hline & OPV7 & $9.5874 \times 10^{-07}$ & $2.73425 \times 10^{-04}$ \\
\hline \multirow{5}{*}{ DPT } & DPT1 & $3.2958 \times 10^{-08}$ & $9.70164 \times 10^{-06}$ \\
\hline & DPT2 & $6.4818 \times 10^{-08}$ & $1.20999 \times 10^{-05}$ \\
\hline & DPT3 & $9.4344 \times 10^{-08}$ & $1.32949 \times 10^{-05}$ \\
\hline & DPT4 & $6.4134 \times 10^{-07}$ & $1.03118 \times 10^{-04}$ \\
\hline & DPT5 & $9.8328 \times 10^{-07}$ & $2.75069 \times 10^{-04}$ \\
\hline \multirow{2}{*}{ Hepatitis B } & НерВ1 & $1.1741 \times 10^{-06}$ & $2.7177 \times 10^{-04}$ \\
\hline & НерВ2 & $1.1741 \times 10^{-06}$ & $2.71907 \times 10^{-04}$ \\
\hline \multirow{3}{*}{ Hib } & Hib1 & $1.2841 \times 10^{-06}$ & $2.72553 \times 10^{-04}$ \\
\hline & Hib2 & $1.2814 \times 10^{-06}$ & $2.72303 \times 10^{-04}$ \\
\hline & Hib3 & $1.305 \times 10^{-06}$ & $2.72429 \times 10^{-04}$ \\
\hline \multicolumn{2}{|c|}{ Measles } & $1.6381 \times 10^{-06}$ & $2.53068 \times 10^{-04}$ \\
\hline \multicolumn{2}{|c|}{ MMR } & $1.702 \times 10^{-06}$ & $2.75305 \times 10^{-04}$ \\
\hline
\end{tabular}


Table 3 represents estimates of design effect of proportion of children vaccinated against different types of vaccines. Design effect estimates are calculated for two stage cluster sampling vs simple random sampling, systematic sampling vs simple random sampling and cluster sampling vs systematic sampling. It is seen that design effect estimates are high in systematic sampling vs simple random sampling rather than the two stage cluster sampling vs simple random sampling and cluster sampling vs systematic sampling for all the vaccines considered here.

To study the homogeneity of clusters chi-square test has been performed. Here calculated value of $\chi^{2}$ is 116.68 with 29 d.f. and p value is 0.00 that is the test statistic is significant and we reject the null hypothesis and concluded that the proportions of children vaccinated against Hepatitis B (at birth) are not equal. Let us start with computing all the proportions of children vaccinated against Hepatitis B (at birth) (given in Table 4).

Table 3. Estimates of design effect of proportion of children vaccinated.

\begin{tabular}{|c|c|c|c|c|}
\hline \multirow{2}{*}{\multicolumn{2}{|c|}{ Vaccine }} & \multicolumn{3}{|c|}{ Design effect } \\
\hline & & Cluster vs SRS & Systematic vs SRS & Cluster vs systematic \\
\hline \multicolumn{2}{|c|}{ BCG } & 0.000835516 & 0.221728395 & 0.003768 \\
\hline \multirow{7}{*}{ OPV } & OPV1 & 0.001084923 & 0.442469136 & 0.002452 \\
\hline & OPV2 & 0.001511716 & 0.880987654 & 0.003397 \\
\hline & OPV3 & 0.0014739 & 1.207284 & 0.002417 \\
\hline & OPV4 & 0.003949769 & 1.423580247 & 0.008155 \\
\hline & OPV5 & 0.022256 & 0.933037 & 0.023834 \\
\hline & OPV6 & 0.005094 & 0.831239 & 0.005634 \\
\hline & OPV7 & 0.001308 & 0.411678 & 0.003506 \\
\hline \multirow{5}{*}{ DPT } & DPT1 & 0.001511716 & 0.880987654 & 0.003397 \\
\hline & DPT2 & 0.002973041 & 1.098765432 & 0.005357 \\
\hline & DPT3 & 0.002914598 & 1.207283951 & 0.007096 \\
\hline & DPT4 & 0.0054443 & 0.87535464 & 0.00622 \\
\hline & DPT5 & 0.00133547 & 0.41166791 & 0.003575 \\
\hline \multirow{2}{*}{ Hepatitis B } & НерВ1 & 0.004333 & 0.991563 & 0.00432 \\
\hline & НерВ2 & 0.004364 & 0.992063 & 0.004318 \\
\hline \multirow{3}{*}{ Hib } & Hib1 & 0.004710096 & 0.99 & 0.004712 \\
\hline & Hib2 & 0.0047 & 0.989091 & 0.004706 \\
\hline & Hib3 & 0.0047867 & 0.9895506 & 0.00479 \\
\hline \multicolumn{2}{|c|}{ Measles } & 0.0059581 & 0.9380207 & 0.006473 \\
\hline \multicolumn{2}{|c|}{ MMR } & 0.0052098 & 0.8413334 & 0.006182 \\
\hline
\end{tabular}

Table 4. Estimated proportions of children vaccinated against Hepatitis B (at birth).

\begin{tabular}{cccc}
\hline Sl. No. & Ward No. & \multicolumn{2}{c}{ Estimated proportions } \\
\hline 1 & 2 & $p_{1}$ & 0.17 \\
2 & 4 & $p_{2}$ & 0.70 \\
3 & 5 & $p_{3}$ & 0.23 \\
4 & 11 & $p_{4}$ & 0.93 \\
5 & 12 & $p_{5}$ & 0.53 \\
6 & 15 & $p_{6}$ & 0.60 \\
7 & 17 & $p_{7}$ & 0.47 \\
8 & 18 & $p_{8}$ & 0.70 \\
9 & 24 & $p_{9}$ & 0.17 \\
\hline
\end{tabular}




\begin{tabular}{cccc} 
Continued & & & \\
\hline 10 & 25 & $p_{10}$ & 0.67 \\
11 & 26 & $p_{11}$ & 0.63 \\
12 & 33 & $p_{12}$ & 0.73 \\
13 & 35 & $p_{13}$ & 0.40 \\
14 & 36 & $p_{14}$ & 0.63 \\
15 & 37 & $p_{15}$ & 0.63 \\
16 & 38 & $p_{16}$ & 0.43 \\
17 & $p_{17}$ & 0.53 \\
18 & 40 & $p_{18}$ & 0.73 \\
19 & 42 & $p_{19}$ & 0.67 \\
20 & 43 & $p_{20}$ & 0.53 \\
21 & 46 & $p_{21}$ & 0.57 \\
22 & 47 & $p_{22}$ & 0.60 \\
23 & 48 & $p_{23}$ & 0.80 \\
24 & 50 & $p_{24}$ & 0.63 \\
25 & 51 & $p_{25}$ & 0.67 \\
26 & 53 & $p_{26}$ & 0.63 \\
27 & 54 & $p_{27}$ & 0.37 \\
28 & 50 & $p_{28}$ & 0.70 \\
29 & 55 & $p_{29}$ & 0.57 \\
30 & 59 & $p_{30}$ & 0.83 \\
\hline
\end{tabular}

It is seen that Hepatitis B (at birth) vaccine coverage is higher for ward number $11\left(p_{4}=0.93\right.$ ) than all other wards. After that $\left|p_{i}-p_{j}\right|$ and $C V_{i j}$ are computed and compared each of test statistics with the corresponding critical value $C V_{i j}$ (given in Table 5).

Results are significant only for proportion of Hepatitis B (at birth) vaccine coverage for ward number 1 vs ward number 4 ( $p_{1}$ vs $p_{4}$ ), ward number 1 vs ward number 30 ( $p_{1}$ vs $p_{30}$ ), ward number 3 vs ward number $4\left(p_{3}\right.$ vs $\left.p_{4}\right)$, ward number 4 vs ward number $9\left(p_{4}\right.$ vs $\left.p_{9}\right)$ and ward number 9 vs ward number $30\left(p_{9}\right.$ vs $\left.p_{30}\right)$. That is these proportions are not equal. Out of 435 pairs of proportions of vaccination coverage only 5 pairs of proportions are unequal.

\section{Discussion}

Estimates of variances and design effect have been used by Milligan et al. [4] to compare two cluster sampling methods for health surveys in developing countries. Both the methods gave very similar point estimates of vaccination coverage. The estimates of the proportion fully vaccinated were 0.56 (EPI) and 0.54 (segmented method) and suggest that EPI method can give accurate and precise results. On the basis of this previous study the current study tries to estimate the design effect of vaccination coverage of the considered study population. In a study of comparison of survey methodologies relative feasibility of the sampling methodologies was assessed by Luman et al. [3]. Coverage with routine vaccinations among children aged 12 - 23 months was much lower than coverage achieved through the measles SIA (supplemental immunization activities). Also Katz et al. studied bias estimate and design effects associated with the EPI sampling design [11]. Brogan et al. suggested techniques for improving the accuracy of the EPI cluster survey method [12]. In Bangladesh overall only $64.1 \%$ of children received the measles vaccine, polio1 has the highest coverage rate in both urban and rural areas. The study also reported that percentage of receiving DPT and polio vaccine decreases when higher doses are given [13]. Chhabra et al. studied the factors affecting the vaccination coverage in two urbanized villages of East Delhi. The coverage levels were highest for BCG (82.7\%) and DPT/OPV1 (81.5\%) and lowest for HBV3 (24.3\%). About $65.3 \%$ had received primary immunization while only $41.6 \%$ of children had received MMR vaccine [14]. 
D. C. Nath, B. Patowari

Table 5. Pairwise Comparison of test statistics $\left(\left|p_{i}-p_{j}\right|\right)$ and critical values $\left(C V_{i j}\right)$.

\begin{tabular}{|c|c|c|c|c|c|c|c|c|c|c|c|}
\hline \multirow{2}{*}{$\begin{array}{c}\text { SI No. } \\
1\end{array}$} & \multicolumn{2}{|c|}{$\left|\boldsymbol{p}_{i}-\boldsymbol{p}_{j}\right|$} & \multirow{2}{*}{$\begin{array}{l}C \boldsymbol{V}_{\boldsymbol{i j}} \\
0.71\end{array}$} & \multirow{2}{*}{\begin{tabular}{|c|} 
SI No. \\
146
\end{tabular}} & \multicolumn{2}{|c|}{$\left|\boldsymbol{p}_{i}-\boldsymbol{p}_{j}\right|$} & \multirow{2}{*}{$\begin{array}{l}C \boldsymbol{V}_{i j} \\
0.83\end{array}$} & \multirow{2}{*}{\begin{tabular}{|c|} 
SI No. \\
291 \\
\end{tabular}} & \multicolumn{2}{|c|}{$\left|p_{i}-p_{j}\right|$} & \multirow{2}{*}{$\begin{array}{l}\boldsymbol{C} \boldsymbol{V}_{\boldsymbol{i j}} \\
0.83\end{array}$} \\
\hline & $p_{1}-p_{2}$ & 0.53 & & & $p_{6}-p_{17}$ & 0.07 & & & $p_{13}-p_{22}$ & 0.20 & \\
\hline 2 & $p_{1}-p_{3}$ & 0.06 & 0.67 & 147 & $p_{6}-p_{18}$ & 0.13 & 0.79 & 292 & $p_{13}-p_{23}$ & 0.40 & 0.75 \\
\hline 3 & $p_{1}-p_{4}{ }^{*}$ & 0.76 & 0.54 & 148 & $p_{6}-p_{19}$ & 0.07 & 0.81 & 293 & $p_{13}-p_{24}$ & 0.23 & 0.82 \\
\hline 4 & $p_{1}-p_{5}$ & 0.36 & 0.74 & 149 & $p_{6}-p_{20}$ & 0.07 & 0.83 & 294 & $p_{13}-p_{25}$ & 0.27 & 0.81 \\
\hline 5 & $p_{1}-p_{6}$ & 0.43 & 0.74 & 150 & $p_{6}-p_{21}$ & 0.03 & 0.83 & 295 & $p_{13}-p_{26}$ & 0.23 & 0.82 \\
\hline 6 & $p_{1}-p_{7}$ & 0.30 & 0.74 & 151 & $p_{6}-p_{22}$ & 0.00 & 0.83 & 296 & $p_{13}-p_{27}$ & 0.03 & 0.82 \\
\hline 7 & $p_{1}-p_{8}$ & 0.53 & 0.71 & 152 & $p_{6}-p_{23}$ & 0.20 & 0.75 & 297 & $p_{13}-p_{28}$ & 0.30 & 0.80 \\
\hline 8 & $p_{1}-p_{9}$ & 0.00 & 0.63 & 153 & $p_{6}-p_{24}$ & 0.03 & 0.82 & 298 & $p_{13}-p_{29}$ & 0.17 & 0.83 \\
\hline 9 & $p_{1}-p_{10}$ & 0.50 & 0.72 & 154 & $p_{6}-p_{25}$ & 0.07 & 0.81 & 299 & $p_{13}-p_{30}$ & 0.43 & 0.74 \\
\hline 10 & $p_{1}-p_{11}$ & 0.46 & 0.73 & 155 & $p_{6}-p_{26}$ & 0.03 & 0.82 & 300 & $p_{14}-p_{15}$ & 0.00 & 0.81 \\
\hline 11 & $p_{1}-p_{12}$ & 0.56 & 0.69 & 156 & $p_{6}-p_{27}$ & 0.23 & 0.82 & 301 & $p_{14}-p_{16}$ & 0.20 & 0.82 \\
\hline 12 & $p_{1}-p_{13}$ & 0.23 & 0.74 & 157 & $p_{6}-p_{28}$ & 0.10 & 0.80 & 302 & $p_{14}-p_{17}$ & 0.10 & 0.83 \\
\hline 13 & $p_{1}-p_{14}$ & 0.46 & 0.73 & 158 & $p_{6}-p_{29}$ & 0.03 & 0.83 & 303 & $p_{14}-p_{18}$ & 0.10 & 0.78 \\
\hline 14 & $p_{1}-p_{15}$ & 0.46 & 0.73 & 159 & $p_{6}-p_{30}$ & 0.23 & 0.74 & 304 & $p_{14}-p_{19}$ & 0.04 & 0.80 \\
\hline 15 & $p_{1}-p_{16}$ & 0.26 & 0.74 & 160 & $p_{7}-p_{8}$ & 0.23 & 0.81 & 305 & $p_{14}-p_{20}$ & 0.10 & 0.83 \\
\hline 16 & $p_{1}-p_{17}$ & 0.36 & 0.74 & 161 & $p_{7}-p_{9}$ & 0.30 & 0.74 & 306 & $p_{14}-p_{21}$ & 0.06 & 0.82 \\
\hline 17 & $p_{1}-p_{18}$ & 0.56 & 0.69 & 162 & $p_{7}-p_{10}$ & 0.20 & 0.82 & 307 & $p_{14}-p_{22}$ & 0.03 & 0.82 \\
\hline 18 & $p_{1}-p_{19}$ & 0.50 & 0.72 & 163 & $p_{7}-p_{11}$ & 0.16 & 0.83 & 308 & $p_{14}-p_{23}$ & 0.17 & 0.75 \\
\hline 19 & $p_{1}-p_{20}$ & 0.36 & 0.74 & 164 & $p_{7}-p_{12}$ & 0.26 & 0.80 & 309 & $p_{14}-p_{24}$ & 0.00 & 0.81 \\
\hline 20 & $p_{1}-p_{21}$ & 0.40 & 0.74 & 165 & $p_{7}-p_{13}$ & 0.07 & 0.83 & 310 & $p_{14}-p_{25}$ & 0.04 & 0.80 \\
\hline 21 & $p_{1}-p_{22}$ & 0.43 & 0.74 & 166 & $p_{7}-p_{14}$ & 0.16 & 0.83 & 311 & $p_{14}-p_{26}$ & 0.00 & 0.81 \\
\hline 22 & $p_{1}-p_{23}$ & 0.63 & 0.65 & 167 & $p_{7}-p_{15}$ & 0.16 & 0.83 & 312 & $p_{14}-p_{27}$ & 0.26 & 0.81 \\
\hline 23 & $p_{1}-p_{24}$ & 0.46 & 0.73 & 168 & $p_{7}-p_{16}$ & 0.04 & 0.84 & 313 & $p_{14}-p_{28}$ & 0.07 & 0.79 \\
\hline 24 & $p_{1}-p_{25}$ & 0.50 & 0.72 & 169 & $p_{7}-p_{17}$ & 0.06 & 0.84 & 314 & $p_{14}-p_{29}$ & 0.06 & 0.82 \\
\hline 25 & $p_{1}-p_{26}$ & 0.46 & 0.73 & 170 & $p_{7}-p_{18}$ & 0.26 & 0.80 & 315 & $p_{14}-p_{30}$ & 0.20 & 0.73 \\
\hline 26 & $p_{1}-p_{27}$ & 0.20 & 0.73 & 171 & $p_{7}-p_{19}$ & 0.20 & 0.82 & 316 & $p_{15}-p_{16}$ & 0.20 & 0.82 \\
\hline 27 & $p_{1}-p_{28}$ & 0.53 & 0.71 & 172 & $p_{7}-p_{20}$ & 0.06 & 0.84 & 317 & $p_{15}-p_{17}$ & 0.10 & 0.83 \\
\hline 28 & $p_{1}-p_{29}$ & 0.40 & 0.74 & 173 & $p_{7}-p_{21}$ & 0.10 & 0.84 & 318 & $p_{15}-p_{18}$ & 0.10 & 0.78 \\
\hline 29 & $p_{1}-p_{30}{ }^{*}$ & 0.66 & 0.63 & 174 & $p_{7}-p_{22}$ & 0.13 & 0.83 & 319 & $p_{15}-p_{19}$ & 0.04 & 0.80 \\
\hline 30 & $p_{2}-p_{3}$ & 0.47 & 0.74 & 175 & $p_{7}-p_{23}$ & 0.33 & 0.76 & 320 & $p_{15}-p_{20}$ & 0.10 & 0.83 \\
\hline 31 & $p_{2}-p_{4}$ & 0.23 & 0.62 & 176 & $p_{7}-p_{24}$ & 0.16 & 0.83 & 321 & $p_{15}-p_{21}$ & 0.06 & 0.82 \\
\hline 32 & $p_{2}-p_{5}$ & 0.17 & 0.81 & 177 & $p_{7}-p_{25}$ & 0.20 & 0.82 & 322 & $p_{15}-p_{22}$ & 0.03 & 0.82 \\
\hline 33 & $p_{2}-p_{6}$ & 0.10 & 0.80 & 178 & $p_{7}-p_{26}$ & 0.16 & 0.83 & 323 & $p_{15}-p_{23}$ & 0.17 & 0.75 \\
\hline 34 & $p_{2}-p_{7}$ & 0.23 & 0.81 & 179 & $p_{7}-p_{27}$ & 0.10 & 0.83 & 324 & $p_{15}-p_{24}$ & 0.00 & 0.81 \\
\hline 35 & $p_{2}-p_{8}$ & 0.00 & 0.77 & 180 & $p_{7}-p_{28}$ & 0.23 & 0.81 & 325 & $p_{15}-p_{25}$ & 0.04 & 0.80 \\
\hline 36 & $p_{2}-p_{9}$ & 0.53 & 0.71 & 181 & $p_{7}-p_{29}$ & 0.10 & 0.84 & 326 & $p_{15}-p_{26}$ & 0.00 & 0.81 \\
\hline 37 & $p_{2}-p_{10}$ & 0.03 & 0.78 & 182 & $p_{7}-p_{30}$ & 0.36 & 0.74 & 327 & $p_{15}-p_{27}$ & 0.26 & 0.81 \\
\hline 38 & $p_{2}-p_{11}$ & 0.07 & 0.79 & 183 & $p_{8}-p_{9}$ & 0.53 & 0.71 & 328 & $p_{15}-p_{28}$ & 0.07 & 0.79 \\
\hline 39 & $p_{2}-p_{12}$ & 0.03 & 0.76 & 184 & $p_{8}-p_{10}$ & 0.03 & 0.78 & 329 & $p_{15}-p_{29}$ & 0.06 & 0.82 \\
\hline 40 & $p_{2}-p_{13}$ & 0.30 & 0.80 & 185 & $p_{8}-p_{11}$ & 0.07 & 0.79 & 330 & $p_{15}-p_{30}$ & 0.20 & 0.73 \\
\hline 41 & $p_{2}-p_{14}$ & 0.07 & 0.79 & 186 & $p_{8}-p_{12}$ & 0.03 & 0.76 & 331 & $p_{16}-p_{17}$ & 0.10 & 0.84 \\
\hline 42 & $p_{2}-p_{15}$ & 0.07 & 0.79 & 187 & $p_{8}-p_{13}$ & 0.30 & 0.80 & 332 & $p_{16}-p_{18}$ & 0.30 & 0.79 \\
\hline 43 & $p_{2}-p_{16}$ & 0.27 & 0.80 & 188 & $p_{8}-p_{14}$ & 0.07 & 0.79 & 333 & $p_{16}-p_{19}$ & 0.24 & 0.81 \\
\hline
\end{tabular}




\section{Continued}

\begin{tabular}{|c|c|c|c|c|c|c|c|c|c|c|c|}
\hline 44 & $p_{2}-p_{17}$ & 0.17 & 0.81 & 189 & $p_{8}-p_{15}$ & 0.07 & 0.79 & 334 & $p_{16}-p_{20}$ & 0.10 & 0.84 \\
\hline 45 & $p_{2}-p_{18}$ & 0.03 & 0.76 & 190 & $p_{8}-p_{16}$ & 0.27 & 0.80 & 335 & $p_{16}-p_{21}$ & 0.14 & 0.83 \\
\hline 46 & $p_{2}-p_{19}$ & 0.03 & 0.78 & 191 & $p_{8}-p_{17}$ & 0.17 & 0.81 & 336 & $p_{16}-p_{22}$ & 0.17 & 0.83 \\
\hline 47 & $p_{2}-p_{20}$ & 0.17 & 0.81 & 192 & $p_{8}-p_{18}$ & 0.03 & 0.76 & 337 & $p_{16}-p_{23}$ & 0.37 & 0.76 \\
\hline 48 & $p_{2}-p_{21}$ & 0.13 & 0.80 & 193 & $p_{8}-p_{19}$ & 0.03 & 0.78 & 338 & $p_{16}-p_{24}$ & 0.20 & 0.82 \\
\hline 49 & $p_{2}-p_{22}$ & 0.10 & 0.80 & 194 & $p_{8}-p_{20}$ & 0.17 & 0.81 & 339 & $p_{16}-p_{25}$ & 0.24 & 0.81 \\
\hline 50 & $p_{2}-p_{23}$ & 0.10 & 0.72 & 195 & $p_{8}-p_{21}$ & 0.13 & 0.80 & 340 & $p_{16}-p_{26}$ & 0.20 & 0.82 \\
\hline 51 & $p_{2}-p_{24}$ & 0.07 & 0.79 & 196 & $p_{8}-p_{22}$ & 0.10 & 0.80 & 341 & $p_{16}-p_{27}$ & 0.06 & 0.82 \\
\hline 52 & $p_{2}-p_{25}$ & 0.03 & 0.78 & 197 & $p_{8}-p_{23}$ & 0.10 & 0.72 & 342 & $p_{16}-p_{28}$ & 0.27 & 0.80 \\
\hline 53 & $p_{2}-p_{26}$ & 0.07 & 0.79 & 198 & $p_{8}-p_{24}$ & 0.07 & 0.79 & 343 & $p_{16}-p_{29}$ & 0.14 & 0.83 \\
\hline 54 & $p_{2}-p_{27}$ & 0.33 & 0.79 & 199 & $p_{8}-p_{25}$ & 0.03 & 0.78 & 344 & $p_{16}-p_{30}$ & 0.40 & 0.74 \\
\hline 55 & $p_{2}-p_{28}$ & 0.00 & 0.77 & 200 & $p_{8}-p_{26}$ & 0.07 & 0.79 & 345 & $p_{17}-p_{18}$ & 0.20 & 0.80 \\
\hline 56 & $p_{2}-p_{29}$ & 0.13 & 0.80 & 201 & $p_{8}-p_{27}$ & 0.33 & 0.79 & 346 & $p_{17}-p_{19}$ & 0.14 & 0.82 \\
\hline 57 & $p_{2}-p_{30}$ & 0.13 & 0.71 & 202 & $p_{8}-p_{28}$ & 0.00 & 0.77 & 347 & $p_{17}-p_{20}$ & 0.00 & 0.84 \\
\hline 58 & $p_{3}-p_{4}{ }^{*}$ & 0.70 & 0.59 & 203 & $p_{8}-p_{29}$ & 0.13 & 0.80 & 348 & $p_{17}-p_{21}$ & 0.04 & 0.84 \\
\hline 59 & $p_{3}-p_{5}$ & 0.30 & 0.78 & 204 & $p_{8}-p_{30}$ & 0.13 & 0.71 & 349 & $p_{17}-p_{22}$ & 0.07 & 0.83 \\
\hline 60 & $p_{3}-p_{6}$ & 0.37 & 0.77 & 205 & $p_{9}-p_{10}$ & 0.50 & 0.72 & 350 & $p_{17}-p_{23}$ & 0.27 & 0.76 \\
\hline 61 & $p_{3}-p_{7}$ & 0.24 & 0.78 & 206 & $p_{9}-p_{11}$ & 0.46 & 0.73 & 351 & $p_{17}-p_{24}$ & 0.10 & 0.83 \\
\hline 62 & $p_{3}-p_{8}$ & 0.47 & 0.74 & 207 & $p_{9}-p_{12}$ & 0.56 & 0.69 & 352 & $p_{17}-p_{25}$ & 0.14 & 0.82 \\
\hline 63 & $p_{3}-p_{9}$ & 0.06 & 0.67 & 208 & $p_{9}-p_{13}$ & 0.23 & 0.74 & 353 & $p_{17}-p_{26}$ & 0.10 & 0.83 \\
\hline 64 & $p_{3}-p_{10}$ & 0.44 & 0.75 & 209 & $p_{9}-p_{14}$ & 0.46 & 0.73 & 354 & $p_{17}-p_{27}$ & 0.16 & 0.83 \\
\hline 65 & $p_{3}-p_{11}$ & 0.40 & 0.76 & 210 & $p_{9}-p_{15}$ & 0.46 & 0.73 & 355 & $p_{17}-p_{28}$ & 0.17 & 0.81 \\
\hline 66 & $p_{3}-p_{12}$ & 0.50 & 0.73 & 211 & $p_{9}-p_{16}$ & 0.26 & 0.74 & 356 & $p_{17}-p_{29}$ & 0.04 & 0.84 \\
\hline 67 & $p_{3}-p_{13}$ & 0.17 & 0.77 & 212 & $p_{9}-p_{17}$ & 0.36 & 0.74 & 357 & $p_{17}-p_{30}$ & 0.30 & 0.74 \\
\hline 68 & $p_{3}-p_{14}$ & 0.40 & 0.76 & 213 & $p_{9}-p_{18}$ & 0.56 & 0.69 & 358 & $p_{18}-p_{19}$ & 0.06 & 0.77 \\
\hline 69 & $p_{3}-p_{15}$ & 0.40 & 0.76 & 214 & $p_{9}-p_{19}$ & 0.50 & 0.72 & 359 & $p_{18}-p_{20}$ & 0.20 & 0.80 \\
\hline 70 & $p_{3}-p_{16}$ & 0.20 & 0.77 & 215 & $p_{9}-p_{20}$ & 0.36 & 0.74 & 360 & $p_{18}-p_{21}$ & 0.16 & 0.79 \\
\hline 71 & $p_{3}-p_{17}$ & 0.30 & 0.78 & 216 & $p_{9}-p_{21}$ & 0.40 & 0.74 & 361 & $p_{18}-p_{22}$ & 0.13 & 0.79 \\
\hline 72 & $p_{3}-p_{18}$ & 0.50 & 0.73 & 217 & $p_{9}-p_{22}$ & 0.43 & 0.74 & 362 & $p_{18}-p_{23}$ & 0.07 & 0.71 \\
\hline 73 & $p_{3}-p_{19}$ & 0.44 & 0.75 & 218 & $p_{9}-p_{23}$ & 0.63 & 0.65 & 363 & $p_{18}-p_{24}$ & 0.10 & 0.78 \\
\hline 74 & $p_{3}-p_{20}$ & 0.30 & 0.78 & 219 & $p_{9}-p_{24}$ & 0.46 & 0.73 & 364 & $p_{18}-p_{25}$ & 0.06 & 0.77 \\
\hline 75 & $p_{3}-p_{21}$ & 0.34 & 0.77 & 220 & $p_{9}-p_{25}$ & 0.50 & 0.72 & 365 & $p_{18}-p_{26}$ & 0.10 & 0.78 \\
\hline 76 & $p_{3}-p_{22}$ & 0.37 & 0.77 & 221 & $p_{9}-p_{26}$ & 0.46 & 0.73 & 366 & $p_{18}-p_{27}$ & 0.36 & 0.78 \\
\hline 77 & $p_{3}-p_{23}$ & 0.57 & 0.69 & 222 & $p_{9}-p_{27}$ & 0.20 & 0.73 & 367 & $p_{18}-p_{28}$ & 0.03 & 0.76 \\
\hline 78 & $p_{3}-p_{24}$ & 0.40 & 0.76 & 223 & $p_{9}-p_{28}$ & 0.53 & 0.71 & 368 & $p_{18}-p_{29}$ & 0.16 & 0.79 \\
\hline 79 & $p_{3}-p_{25}$ & 0.44 & 0.75 & 224 & $p_{9}-p_{29}$ & 0.40 & 0.74 & 369 & $p_{18}-p_{30}$ & 0.10 & 0.69 \\
\hline 80 & $p_{3}-p_{26}$ & 0.40 & 0.76 & 225 & $p_{9}-p_{30}{ }^{*}$ & 0.66 & 0.63 & 370 & $p_{19}-p_{20}$ & 0.14 & 0.82 \\
\hline 81 & $p_{3}-p_{27}$ & 0.14 & 0.76 & 226 & $p_{10}-p_{11}$ & 0.04 & 0.80 & 371 & $p_{19}-p_{21}$ & 0.10 & 0.81 \\
\hline 82 & $p_{3}-p_{28}$ & 0.47 & 0.74 & 227 & $p_{10}-p_{12}$ & 0.06 & 0.77 & 372 & $p_{19}-p_{22}$ & 0.07 & 0.81 \\
\hline 83 & $p_{3}-p_{29}$ & 0.34 & 0.77 & 228 & $p_{10}-p_{13}$ & 0.27 & 0.81 & 373 & $p_{19}-p_{23}$ & 0.13 & 0.74 \\
\hline 84 & $p_{3}-p_{30}$ & 0.60 & 0.67 & 229 & $p_{10}-p_{14}$ & 0.04 & 0.80 & 374 & $p_{19}-p_{24}$ & 0.04 & 0.80 \\
\hline 85 & $p_{4}-P_{p}$ & 0.40 & 0.67 & 230 & $p_{10}-p_{15}$ & 0.04 & 0.80 & 375 & $p_{19}-p_{25}$ & 0.00 & 0.79 \\
\hline 86 & $p_{4}-p_{6}$ & 0.33 & 0.66 & 231 & $p_{10}-p_{16}$ & 0.24 & 0.81 & 376 & $p_{19}-p_{26}$ & 0.04 & 0.80 \\
\hline 87 & $p_{4}-p_{7}$ & 0.46 & 0.67 & 232 & $p_{10}-p_{17}$ & 0.14 & 0.82 & 377 & $p_{19}-p_{27}$ & 0.30 & 0.80 \\
\hline
\end{tabular}


D. C. Nath, B. Patowari

\section{Continued}

\begin{tabular}{|c|c|c|c|c|c|c|c|c|c|c|c|}
\hline 88 & $p_{4}-p_{8}$ & 0.23 & 0.62 & 233 & $p_{10}-p_{18}$ & 0.06 & 0.77 & 378 & $p_{19}-p_{28}$ & 0.03 & 0.78 \\
\hline 89 & $p_{4}-p_{9}{ }^{*}$ & 0.76 & 0.54 & 234 & $p_{10}-p_{19}$ & 0.00 & 0.79 & 379 & $p_{19}-p_{29}$ & 0.10 & 0.81 \\
\hline 90 & $p_{4}-p_{10}$ & 0.26 & 0.64 & 235 & $p_{10}-p_{20}$ & 0.14 & 0.82 & 380 & $p_{19}-p_{30}$ & 0.16 & 0.72 \\
\hline 91 & $p_{4}-p_{11}$ & 0.30 & 0.65 & 236 & $p_{10}-p_{21}$ & 0.10 & 0.81 & 381 & $p_{20}-p_{21}$ & 0.04 & 0.84 \\
\hline 92 & $p_{4}-p_{12}$ & 0.20 & 0.61 & 237 & $p_{10}-p_{22}$ & 0.07 & 0.81 & 382 & $p_{20}-p_{22}$ & 0.07 & 0.83 \\
\hline 93 & $p_{4}-p_{13}$ & 0.53 & 0.66 & 238 & $p_{10}-p_{23}$ & 0.13 & 0.74 & 383 & $p_{20}-p_{23}$ & 0.27 & 0.76 \\
\hline 94 & $p_{4}-p_{14}$ & 0.30 & 0.65 & 239 & $p_{10}-p_{24}$ & 0.04 & 0.80 & 384 & $p_{20}-p_{24}$ & 0.10 & 0.83 \\
\hline 95 & $p_{4}-p_{15}$ & 0.30 & 0.65 & 240 & $p_{10}-p_{25}$ & 0.00 & 0.79 & 385 & $p_{20}-p_{25}$ & 0.14 & 0.82 \\
\hline 96 & $p_{4}-p_{16}$ & 0.50 & 0.66 & 241 & $p_{10}-p_{26}$ & 0.04 & 0.80 & 386 & $p_{20}-p_{26}$ & 0.10 & 0.83 \\
\hline 97 & $p_{4}-p_{17}$ & 0.40 & 0.67 & 242 & $p_{10}-p_{27}$ & 0.30 & 0.80 & 387 & $p_{20}-p_{27}$ & 0.16 & 0.83 \\
\hline 98 & $p_{4}-p_{18}$ & 0.20 & 0.61 & 243 & $p_{10}-p_{28}$ & 0.03 & 0.78 & 388 & $p_{20}-p_{28}$ & 0.17 & 0.81 \\
\hline 99 & $p_{4}-p_{19}$ & 0.26 & 0.64 & 244 & $p_{10}-p_{29}$ & 0.10 & 0.81 & 389 & $p_{20}-p_{29}$ & 0.04 & 0.84 \\
\hline 100 & $p_{4}-p_{20}$ & 0.40 & 0.67 & 245 & $p_{10}-p_{30}$ & 0.16 & 0.72 & 390 & $p_{20}-p_{30}$ & 0.30 & 0.74 \\
\hline 101 & $p_{4}-p_{21}$ & 0.36 & 0.66 & 246 & $p_{11}-p_{12}$ & 0.10 & 0.78 & 391 & $p_{21}-p_{22}$ & 0.03 & 0.83 \\
\hline 102 & $p_{4}-p_{22}$ & 0.33 & 0.66 & 247 & $p_{11}-p_{13}$ & 0.23 & 0.82 & 392 & $p_{21}-p_{23}$ & 0.23 & 0.76 \\
\hline 103 & $p_{4}-p_{23}$ & 0.13 & 0.57 & 248 & $p_{11}-p_{14}$ & 0.00 & 0.81 & 393 & $p_{21}-p_{24}$ & 0.06 & 0.82 \\
\hline 104 & $p_{4}-p_{24}$ & 0.30 & 0.65 & 249 & $p_{11}-p_{15}$ & 0.00 & 0.81 & 394 & $p_{21}-p_{25}$ & 0.10 & 0.81 \\
\hline 105 & $p_{4}-p_{25}$ & 0.26 & 0.64 & 250 & $p_{11}-p_{16}$ & 0.20 & 0.82 & 395 & $p_{21}-p_{26}$ & 0.06 & 0.82 \\
\hline 106 & $p_{4}-p_{26}$ & 0.30 & 0.65 & 251 & $p_{11}-p_{17}$ & 0.10 & 0.83 & 396 & $p_{21}-p_{27}$ & 0.20 & 0.82 \\
\hline 107 & $p_{4}-p_{27}$ & 0.56 & 0.65 & 252 & $p_{11}-p_{18}$ & 0.10 & 0.78 & 397 & $p_{21}-p_{28}$ & 0.13 & 0.80 \\
\hline 108 & $p_{4}-p_{28}$ & 0.23 & 0.62 & 253 & $p_{11}-p_{19}$ & 0.04 & 0.80 & 398 & $p_{21}-p_{29}$ & 0.00 & 0.83 \\
\hline 109 & $p_{4}-p_{29}$ & 0.36 & 0.66 & 254 & $p_{11}-p_{20}$ & 0.10 & 0.83 & 399 & $p_{21}-p_{30}$ & 0.26 & 0.74 \\
\hline 110 & $p_{4}-p_{30}$ & 0.10 & 0.54 & 255 & $p_{11}-p_{21}$ & 0.06 & 0.82 & 400 & $p_{22}-p_{23}$ & 0.20 & 0.75 \\
\hline 111 & $p_{5}-p_{6}$ & 0.07 & 0.83 & 256 & $p_{11}-p_{22}$ & 0.03 & 0.82 & 401 & $p_{22}-p_{24}$ & 0.03 & 0.82 \\
\hline 112 & $p_{5}-p_{7}$ & 0.06 & 0.84 & 257 & $p_{11}-p_{23}$ & 0.17 & 0.75 & 402 & $p_{22}-p_{25}$ & 0.07 & 0.81 \\
\hline 113 & $p_{5}-p_{8}$ & 0.17 & 0.81 & 258 & $p_{11}-p_{24}$ & 0.00 & 0.81 & 403 & $p_{22}-p_{26}$ & 0.03 & 0.82 \\
\hline 114 & $p_{5}-p_{9}$ & 0.36 & 0.74 & 259 & $p_{11}-p_{25}$ & 0.04 & 0.80 & 404 & $p_{22}-p_{27}$ & 0.23 & 0.82 \\
\hline 115 & $p_{5}-p_{10}$ & 0.14 & 0.82 & 260 & $p_{11}-p_{26}$ & 0.00 & 0.81 & 405 & $p_{22}-p_{28}$ & 0.10 & 0.80 \\
\hline 116 & $p_{5}-p_{11}$ & 0.10 & 0.83 & 261 & $p_{11}-p_{27}$ & 0.26 & 0.81 & 406 & $p_{22}-p_{29}$ & 0.03 & 0.83 \\
\hline 117 & $p_{5}-p_{12}$ & 0.20 & 0.80 & 262 & $p_{11}-p_{28}$ & 0.07 & 0.79 & 407 & $p_{22}-p_{30}$ & 0.23 & 0.74 \\
\hline 118 & $p_{5}-p_{13}$ & 0.13 & 0.83 & 263 & $p_{11}-p_{29}$ & 0.06 & 0.82 & 408 & $p_{23}-p_{24}$ & 0.17 & 0.75 \\
\hline 119 & $p_{5}-p_{14}$ & 0.10 & 0.83 & 264 & $p_{11}-p_{30}$ & 0.20 & 0.73 & 409 & $p_{23}-p_{25}$ & 0.13 & 0.74 \\
\hline 120 & $p_{5}-p_{15}$ & 0.10 & 0.83 & 265 & $p_{12}-p_{13}$ & 0.33 & 0.79 & 410 & $p_{23}-p_{26}$ & 0.17 & 0.75 \\
\hline 121 & $p_{5}-p_{16}$ & 0.10 & 0.84 & 266 & $p_{12}-p_{14}$ & 0.10 & 0.78 & 411 & $p_{23}-p_{27}$ & 0.43 & 0.75 \\
\hline 122 & $p_{5}-p_{17}$ & 0.00 & 0.84 & 267 & $p_{12}-p_{15}$ & 0.10 & 0.78 & 412 & $p_{23}-p_{28}$ & 0.10 & 0.72 \\
\hline 123 & $p_{5}-p_{18}$ & 0.20 & 0.80 & 268 & $p_{12}-p_{16}$ & 0.30 & 0.79 & 413 & $p_{23}-p_{29}$ & 0.23 & 0.76 \\
\hline 124 & $p_{5}-p_{19}$ & 0.14 & 0.82 & 269 & $p_{12}-p_{17}$ & 0.20 & 0.80 & 414 & $p_{23}-p_{30}$ & 0.03 & 0.65 \\
\hline 125 & $p_{5}-p_{20}$ & 0.00 & 0.84 & 270 & $p_{12}-p_{18}$ & 0.00 & 0.75 & 415 & $p_{24}-p_{25}$ & 0.04 & 0.80 \\
\hline 126 & $p_{5}-p_{21}$ & 0.04 & 0.84 & 271 & $p_{12}-p_{19}$ & 0.06 & 0.77 & 416 & $p_{24}-p_{26}$ & 0.00 & 0.81 \\
\hline 127 & $p_{5}-p_{22}$ & 0.07 & 0.83 & 272 & $p_{12}-p_{20}$ & 0.20 & 0.80 & 417 & $p_{24}-p_{27}$ & 0.26 & 0.81 \\
\hline 128 & $p_{5}-p_{23}$ & 0.27 & 0.76 & 273 & $p_{12}-p_{21}$ & 0.16 & 0.79 & 418 & $p_{24}-p_{28}$ & 0.07 & 0.79 \\
\hline 129 & $p_{5}-p_{24}$ & 0.10 & 0.83 & 274 & $p_{12}-p_{22}$ & 0.13 & 0.79 & 419 & $p_{24}-p_{29}$ & 0.06 & 0.82 \\
\hline 130 & $p_{5}-p_{25}$ & 0.14 & 0.82 & 275 & $p_{12}-p_{23}$ & 0.07 & 0.71 & 420 & $p_{24}-p_{30}$ & 0.20 & 0.73 \\
\hline 131 & $p_{5}-p_{26}$ & 0.10 & 0.83 & 276 & $p_{12}-p_{24}$ & 0.10 & 0.78 & 421 & $p_{25}-p_{26}$ & 0.04 & 0.80 \\
\hline
\end{tabular}




\begin{tabular}{|c|c|c|c|c|c|c|c|c|c|c|c|}
\hline \multicolumn{12}{|c|}{ Continued } \\
\hline 132 & $p_{5}-p_{27}$ & 0.16 & 0.83 & 277 & $p_{12}-p_{25}$ & 0.06 & 0.77 & 422 & $p_{25}-p_{27}$ & 0.30 & 0.80 \\
\hline 133 & $p_{5}-p_{28}$ & 0.17 & 0.81 & 278 & $p_{12}-p_{26}$ & 0.10 & 0.78 & 423 & $p_{25}-p_{28}$ & 0.03 & 0.78 \\
\hline 134 & $p_{5}-p_{29}$ & 0.04 & 0.84 & 279 & $p_{12}-p_{27}$ & 0.36 & 0.78 & 424 & $p_{25}-p_{29}$ & 0.10 & 0.81 \\
\hline 135 & $p_{5}-p_{30}$ & 0.30 & 0.74 & 280 & $p_{12}-p_{28}$ & 0.03 & 0.76 & 425 & $p_{25}-p_{30}$ & 0.16 & 0.72 \\
\hline 136 & $p_{6}-p_{7}$ & 0.13 & 0.83 & 281 & $p_{12}-p_{29}$ & 0.16 & 0.79 & 426 & $p_{26}-p_{27}$ & 0.26 & 0.81 \\
\hline 137 & $p_{6}-p_{8}$ & 0.10 & 0.80 & 282 & $p_{12}-p_{30}$ & 0.10 & 0.69 & 427 & $p_{26}-p_{28}$ & 0.07 & 0.79 \\
\hline 138 & $p_{6}-p_{9}$ & 0.43 & 0.74 & 283 & $p_{13}-p_{14}$ & 0.23 & 0.82 & 428 & $p_{26}-p_{29}$ & 0.06 & 0.82 \\
\hline 139 & $p_{6}-p_{10}$ & 0.07 & 0.81 & 284 & $p_{13}-p_{15}$ & 0.23 & 0.82 & 429 & $p_{26}-p_{30}$ & 0.20 & 0.73 \\
\hline 140 & $p_{6}-p_{11}$ & 0.03 & 0.82 & 285 & $p_{13}-p_{16}$ & 0.03 & 0.83 & 430 & $p_{27}-p_{28}$ & 0.33 & 0.79 \\
\hline 141 & $p_{6}-p_{12}$ & 0.13 & 0.79 & 286 & $p_{13}-p_{17}$ & 0.13 & 0.83 & 431 & $p_{27}-p_{29}$ & 0.20 & 0.82 \\
\hline 142 & $p_{6}-p_{13}$ & 0.20 & 0.83 & 287 & $p_{13}-p_{18}$ & 0.33 & 0.79 & 432 & $p_{27}-p_{30}$ & 0.46 & 0.73 \\
\hline 143 & $p_{6}-p_{14}$ & 0.03 & 0.82 & 288 & $p_{13}-p_{19}$ & 0.27 & 0.81 & 433 & $p_{28}-p_{29}$ & 0.13 & 0.80 \\
\hline 144 & $p_{6}-p_{15}$ & 0.03 & 0.82 & 289 & $p_{13}-p_{20}$ & 0.13 & 0.83 & 434 & $p_{28}-p_{30}$ & 0.13 & 0.71 \\
\hline 145 & $p_{6}-p_{16}$ & 0.17 & 0.83 & 290 & $p_{13}-p_{21}$ & 0.17 & 0.83 & 435 & $p_{29}-p_{30}$ & 0.26 & 0.74 \\
\hline
\end{tabular}

*Significant pair.

In an Urban Area of Meerut 93.25\% of children in community were found to be completely immunized, 5.25\% partially immunized an only $1.5 \%$ non-immunized [15]. In a study Jain et al. mentioned that $28.9 \%$ of children aged 12 - 23 months were fully immunized with BCG, 3 DPT, 3 OPV and Measles vaccines; around 26.5\% had not received even a single vaccine and $44.5 \%$ were found partially immunized. Around $55.95 \%$ of the eligible children were vaccinated for BCG and measles $43.6 \%$. Though nearly $66.8 \%$ were covered with first dose of DPT and OPV but about 33.2\% children dropped out of the third dose of DPT and OPV for various reasons [16]. In an another study in Gujarat coverage for BCG, OPV3, DPT3 \& Measles were 92.04\%, 85.23\%, 83.71\% \& $82.20 \%$ respectively. Although the vaccination coverage shows higher coverage than previous studies, it is still below the minimum targets set as national goal [17]. Immunization status of children and mothers in the northeastern states (except Assam) was evaluated in comparison with data at the national level using a WHO 30-cluster survey methodology. The proportion of children receiving all the vaccinations like BCG, DPT, OPV, measles in north-eastern states were about $51.9 \%$ as against $63.3 \%$ achieved at the all India level [18]. In this current study it has been observed that the fully vaccination coverage in the study population is not so high; it is almost same with the previous study reported by Phukan et al. [7] with a difference of $1.32 \%$ only. The differences between the two survey methods in case of point estimate are not significant and interval estimates has given better estimates in two stage $(30 \times 30)$ cluster sampling. Two stage $(30 \times 30)$ cluster sampling has given better estimate of variance and design effect of vaccination coverage and design effects are less in two stage cluster sampling vs simple random sampling and cluster sampling vs systematic sampling rather than systematic sampling vs simple random sampling. It has been observed that the clusters are homogeneous (since only 5 pairs of proportions are significant).

\section{Conclusion}

The finding of the present study revealed that there are no significant differences between the point estimates obtained under two sampling schemes. But there are differences between estimated variance of proportion of children vaccinated in two sampling methods. Also in case of interval estimation two stage $(30 \times 30)$ cluster sampling has given better intervals than that of under systematic sampling. Vaccination coverage is high for BCG, OPV and DPT vaccine but it is low for Measles, Hepatitis B, Hib and MMR vaccine and the later doses of OPV and DPT vaccine. Finally the two stage cluster $(30 \times 30)$ sampling is more consistent than the systematic sampling as well as simple random sampling for this study population.

\section{Acknowledgements}

The research was supported by the grant (number 69/40/2008-ECD-II) from Indian Council of Medical Research (ICMR), New Delhi and UGC-BSR one time grant (No. F.19-145/2015(BSR)) and provided to the first author. 


\section{Conflict of Interest}

None.

\section{References}

[1] Burton, A., Monasch, R., Lautenbach, B., Gacic-Dobo, M., Neill, M., Karimov, R., Wolfson, L., Jones, G. and Birmingham, M. (2009) WHO and UNICEF Estimates of National Infant Immunization Coverage: Methods and Process. Bulletin of the World Health Organization, 87, 535-541. http://dx.doi.org/10.2471/BLT.08.053819

[2] Turner, A.G., Magnani, R.J. and Shuaib, M. (1996) A Not Quite as Quick Bit Much Cleaner Alternative to the Expanded Programme on Immunization (EPI) Cluster Survey Design. International Journal of Epidemiology, 25, 198203. http://dx.doi.org/10.1093/ije/25.1.198

[3] Luman, E.T., Worku, A., Berhane, Y., Martin, R. and Cairns, L. (2007) Comparison of Two Survey Methodologies to Assess Vaccination Coverage. International Journal of Epidemiology, 36, 633-641. http://dx.doi.org/10.1093/ije/dym025

[4] Milligan, P., Njie, A. and Benneu, S. (2004) Comparison of Two Cluster Sampling Methods for Health Surveys in Developing Countries. International Journal of Epidemiology, 33, 469-476. http://dx.doi.org/10.1093/ije/dyh096

[5] Nath, D.C. and Patowari, B. (2014) Estimation and Comparison of Immunization Coverage under Different Sampling Methods for Health Surveys. International Journal of Population Research, 2014, Article ID: 850479. http://dx.doi.org/10.1155/2014/850479

[6] WHO-SEARO EPI Fact Sheet India, 2011. http://www.searo.who.int/entity/immunization/data/india_epi_factsheet_2011.pdf?ua=1

[7] Phukan, R.K., Barman, M.P. and Mahanta, J. (2009) Factor Associated with Immunization Coverage of Children in Assam, India: Over the First Year of Life. Journal of Tropical Pediatrics, 55, 249-252. http://dx.doi.org/10.1093/tropej/fmn025

[8] Lee, E.S. and Forthofer, R.N. (2006) Analyzing Complex Survey Data. 2nd Edition, Sage Publications, Thousand Oaks.

[9] Levy, P.S. and Lemeshow, S. (2008) Sampling of Populations: Methods and Applications. 4th Edition, John Wiley \& Sons, Hoboken. http://dx.doi.org/10.1002/9780470374597

[10] The Marascuillo Procedure. www.itl.nist.gov/div898/handbook/prc/section4/prc474.htm

[11] Katz, J., Yoon, S.S., Brendel, K. and West Jr., K.P. (1997) Sampling Designs for Xerophthalmia Prevalence Surveys. International Journal of Epidemiology, 26, 1041-1048. http://dx.doi.org/10.1093/ije/26.5.1041

[12] Brogan, D., Flagg, E.W., Deming, M. and Waldman, R. (1994) Increasing the Accuracy of the Expanded Programme on Immunization's Cluster Survey Design. Annals of Epidemiology, 4, 302-311. http://dx.doi.org/10.1016/1047-2797(94)90086-8

[13] Rahman, M. and Obaida-Nasrin, S. (2010) Factors Affecting Acceptance of Complete Immunization Coverage of Children under Five Years in Rural Bangladesh. Salud Pública de México, 52, 134-140. http://dx.doi.org/10.1590/S0036-36342010000200005

[14] Chhabra, P., Nair, P., Gupta, A., Sandhir, M. and Kannan, A.T. (2007) Immunization in Urbanized Villages of Delhi. Indian Journal of Pediatrics, 74, 131-134. http://dx.doi.org/10.1007/s12098-007-0004-3

[15] Chopra, H., Singh, A.K., Singh, J.V., Bhatnagar, M., Garg, S.K. and Bajpai, S.K. (2006-2007) Status of Routine Immunization in an Urban Area of Meerut. Indian Journal of Community Health, 18(2)-19(1), 19-22.

[16] Jain, S.K., Chawla, U., Gupta, N., Gupta, R.S., Venkatesh, S. and Lal, S. (2006) Child Survival and Safe Motherhood Program in Rajasthan. Indian Journal of Pediatrics, 73, 43-47. http://dx.doi.org/10.1007/BF02758259

[17] Sheth, J.K., Trivedi, K.N., Mehta, J.B. and Oza, U.N. (2012) Assessment of Vaccine Coverage by 30 Cluster Sampling Technique in Rural Gandhinagar. Gujarat National Journal of Community Medicine, 3, 496-501.

[18] Yadav, R.J. and Singh, P. (2004) Immunisation of Children and Mothers in Northeastern States. Health and Population-Perspectives and Issues, 27, 185-193. 


\section{Abbreviations}

BCG: Bacillis Calmette-Guerin;

OPV: Oral Polio Vaccine;

DPT: Diphtheria-Tetanus-Pertusis;

MMR: Measles Mumps Rubella;

Hib: Haemophilus influenza type b;

WHO: World Health Organization;

EPI: Expanded Programme on Immunization. 